\title{
A Snapshot of the International Views of the Treatment of Rectal Cancer Patients, a Multi-regional Survey
}

\author{
International Tendencies in Rectal Cancer
}

${ }^{1,2}$ Antonio Caycedo-Marulanda MSc, MD; ${ }^{1}$ Sunil V. Patel MSc, MD; ${ }^{2}$ Chris P. Verschoor PhD; ${ }^{3}$ Johanna P Uscategui RN; ${ }^{4}$ Sami A Chadi MSc, MD; ${ }^{5}$ Gabriela Moeslein MD; ${ }^{6}$ Manish Chand MBBS, MBA, PhD, ${ }^{7}$ Yasuko Maeda MD, M Phil; ${ }^{8}$ John RT Monson MB, MCh; ${ }^{9}$ Steven D Wexner MD, PhD (Hon); ${ }^{10}$ Julio Mayol MD, PhD.

1. Department of surgery, Queens University, Canada.

2. Health Sciences North Research Institute, Sudbury, Canada

3. Colorectal Surgery North, Sudbury, Canada.

4. Department of surgery University Health Network (UHN), Toronto, Canada

5. Department of surgery, University of Witten/Herdecke. Witten, Germany

6. Department of surgery, University College London, London, United Kingdom.

7. Department of Colorectal Surgery, Western General Hospital. Edinburgh, Scotland

8. Center for Colon and Rectal Surgery, Digestive Health and Surgery Institute, AdventHealth Orlando, United States

9. Department of surgery, Cleveland Clinic Florida, Weston, United States.

10. Department of surgery, Hospital Clinico San Carlos, Madrid, Spain.

Corresponding author

Antonio Caycedo-Marulanda MD. MSc

Kingston General Hospital 76 Stuart Street, Kingston, ON K7L 2V7.

ORCID 0000-0003-2387-5304

caycedomd@gmail.com

@ caycedomarula

Word count excluding abstract: (2497) abstract (290)

Key words: Rectal cancer, treatment, variability, snapshot

This manuscript was accepted in abstract form for a Research Forum Podium

Presentation during ASCRS' Annual Scientific Meeting in Boston, MA, June 6 -10, 2019: Quick Shots: Rectal Cancer 


\section{Abstract}

Background: Management of rectal cancer has a number of potentially appropriate alternatives for each patient. Despite acceptance of standards, practices may vary among regions. There is significant paucity of data in this area. The objective was to create a snapshot of the regional differences.

Design: This online survey included 10 questions. Enquiries focused on controversial topics, on surgeon and hospital volume, surgical margins, appropriateness of surgical approaches and techniques, Watch and Wait strategies and total neoadjuvant therapy. Major colorectal surgery societies around the world were asked to invite their members to complete the survey.

Outcomes measures: Frequency of responses across regions within each question was compared by Fisher's exact test.

Results: 753 participants from 60 countries responded. 8 regions were identified, 4 had sufficient representation for comparisons. Similarities and differences in the therapies amongst these regions were identified. Robotic surgery penetrance is higher in North America, watch and wait is more accepted in South America. Patients in Oceania are more likely to be diverted, Europe has more usage of taTME.

Discussion: This online survey was practical as a mean to provide a rapid assessment of the international picture on consistency and variability of rectal cancer patients care, and to potentially identify opportunities to standardized care to patients. Medical surveys have inherent limitations, pertinent to our study is selection bias is one. 
Conclusions: The management of rectal cancer varies among different regions. Identification of differences is important when considering global efforts to improve management and interpret data. 


\section{Introduction}

Total mesorectal excision (TME) is the most influential advancement in the treatment of patients with rectal cancer (1)(2). For early tumors it is the current standard as it is in conjunction with chemo and radiation therapy for locally advanced tumors \{Kapiteijn:2001jt, Sauer:2004fs, Allaix:2013ct, Bujko:2006fc, McQuade:2017gg\}. Survival has considerably improved over the last century. \{Brouwer:2018hf, Lawson:2019cf\}. As therapeutic options have advanced, the complexity of decision making has increased, requiring the involvement of multiple disciplines . \{Smith:2018iz, Lawson:2019uka, GlynneJones:2017gh\}. Those include the use of a watch and wait protocols for potential organ preservation \{Lawson:2019cf\} \{Dossa:2017hh\}, the advancement of minimally invasive approaches \{Fleshman:2015et, Marks:2010ib\}, \{Penna:2016hx, Baik:2007eo\}, as well as the implementation of total neoadjuvant therapy (TNT) \{Cercek:2018bd\}. Sharing of information and discussion at multidisciplinary care conferences (MDTCC) are commonly used methods available at most contemporary centers $(19,20)$. Despite of ample acceptance of therapeutic standards plus wide access to scientific information, practices may vary dramatically among countries. This interesting phenomenon could possibly be due to differences in resource availability, data interpretation, and multiple sociodemographic factors \{Qaderi:2020cg\}.

The objective of the present study was to explore the differences in the current practices of colorectal surgeons regarding management of rectal cancer patients across multiple geographical regions.

\section{Methods}

Internationally, 10 professional colorectal surgical societies accepted to distribute a closed webbased survey (www.surveymonkey.com) among their membership, including: The Association of Coloproctology of Great Britain \& Ireland (ACGBI), The American Society of Colon \& Rectal surgeons (ASCRS), The Canadian Society of Colon and Rectal Surgeons (CSCRS) , La Sociedad Espanola de Coloproctologia, The European Society of Coloproctology (ESCP), Sociedade Brasileira de Coloproctologia, The Colorectal Surgical Society of Australia and New Zealand, La 
Sociedad Argentina de Coloproctologia, La Sociedad Colombiana de Coloproctologia, and The Mediterranean society of Coloproctology.

\section{The Survey}

The framework for concept and content of questions was agreed upon by the authors. A 10question survey was developed, ensuring inclusion of current and controversial topics related to the management of patients with rectal cancer. The survey was developed and distributed in English language format. Information on country and city of origin of respondents was collected and responses were automatically locked after submission to prevented retrospective modifications.

The survey (Appendix. 1) included questions related to the necessary volume for surgeons and hospitals to understand the complexities related to surgical proficiency; surgical margins; approaches and techniques; the use of watch and wait (W\&W) and total neoadjuvant therapy (TNT). The survey was open from June 6th 2019 to September 16th 2019. Societies were asked to send a reminder in the middle of the study period.

Data were collected and analyzed at Health Sciences North Research Institute. Population size of city of origin was used to stratify responses. Ethical approval by the appropriate local research and ethics board was obtained.

\section{Statistical analysis}

Data were summarized as count and frequency of total respondents and response choice within each question according to region. Those regions with fewer than 15 respondents such as Asia, Central America, Caribbean, and Middle East were excluded prior to analysis. Omitted responses (which did not exceed 7 for any given question) were assigned as missing data prior to analysis. To compare the distribution of response choices for each question across all pairs of geographical areas, a Fisher's exact test was used; p-values were corrected for multiple testing within each question using the Benjamini-Hochberg approach for controlling false-discovery rate. We also compared the frequency in which each region selected the most commonly chosen answer for each question using a Fisher's exact test with multiple testing correction. The most 
common answer for each question was that which exhibited the highest average frequency of response across the four regions included for analysis. All analyses were performed using R v3.6.

\section{Results}

753 individuals from 60 different countries replied to our survey, responses ranged from 746 to 753 with some questions being skipped (less than 7 per question). Response time averaged 4 minutes and 8 seconds. Two peaks of participation were evident: on July $1^{\text {st }}(177)$ and August $12^{\text {th }}(234)$ responses, corresponding to the survey-launching and the elicited reminder. $A$ response rate could not be provided due to the inability to determine the exact number of surgeons reached by the survey.

Eight distinctive regions were identified, 4 had enough participants to justify analysis: [North America (NA), South America (SA), Europe (EU) and Oceania (OC)] (Figure 1). Other regions included: Asia, the Middle East, the Caribbean and Central America, which had minimal representation and therefore were excluded. In most regions, participants (47\%) resided in cities with a population of more than 1 million; the only exception was NA where less than $20 \%$ were located in large cities (>1 million). A quarter of the participants from NA are located in cities with less than 100K people. Most surgeons in NA lived in cities with populations between 100K-500K. In EU and SA, over 70\% of participants resided in large urban centers with a population over one million. EU, OC and SA had small proportion of participants from cities with a population of 100K-500K (Figure.1)

Response frequencies are presented in table 2 and results of comparison tests amongst groups in Figure 2. Question one explored the perceived number of annual cases required to be considered a high-volume surgeon. Fifty-two percent of the total participants considered >20 as the minimum and $10 \%$ in EU and SA considered it to be $>50$ cases. The top choice was similar among the 4 regions, showing no statistically significant difference when compared (Figure 2A). However, when assessing the distribution of responses, there was a significant difference: EU was significantly different from NA $(p<0.001), O C(p<0.001)$, and $S A(p<0.01)$ (Figure $2 B)$. 
When asked about the minimum number of patients to be considered a high-volume center, the most common option was $>40$ annually (43\%). The preferred choice was similar across all regions with no significant differences observed, however the distribution of answers among the regions was significantly different $(p<0.05)$ (Figure $2 A-B)$. Question three inquired about which cases should be presented at MDTC and 80\% considered that all cases require discussion. Regarding the preferred option, cases are significantly more likely to be presented in EU than in the other regions. EU was significantly different from NA ( $p<0.001), O C(p<0.01)$, and $S A(p<0.001)$. (Figure 2A)

W\&W was a "never" option for $15 \%$ of participants. In EU and OC, $25 \%$ and $5.8 \%$ of surgeons respectively, would never consider it as a valid alternative either. $34 \%$ of all participants favored its regular application. The highest proportion of users were in SA (39\%), nonetheless there was no significant differences among the regions regarding the top choice. The distribution of responses demonstrated significant differences when EU was compared to all other regions: NA $(p<0.001)$, OC $(p<0.001)$, and SA $(p<0.01)$.

Question five referred to a clinical scenario presenting a female patient with a low rectal cancer and apparent complete clinical response. The most popular answer was to perform a low anterior resection (LAR) as planned (53\%). Both EU and NA showed a high preference for this option (57\% and $65 \%$ respectively). In OC, performing a LAR was almost as popular as opting for $W \& W(48 \%$ vs $47 \%)$. The majority of users from SA elected W\&W (55\%). For the top answer, there was a significant difference when comparing SA to EU $(p<0.001)$ and NA $(p<0.001)$. Even when comparing NA to OC there was a significant difference [65\% vs $48 \%(p<0.05)$ ]. When considering the preferred surgical approach on an obese male with a low T2NO rectal cancer, the most popular choice was laparoscopic surgery (39\%). The top option in NA was robotic surgery [54.1\%]. When comparing the use of the laparoscopic approach in NA (23.9\%) against other regions, significant differences were found (NA. VS. EU [45.8\% (p<00.1)] and SA [51.9\% ( $p<0.001)])$; conversely, there was no difference between NA and OC ( $p>0.05)$. 
Transanal total mesorectal excision (taTME) was the most popular approach in EU (33.5\%). For distribution of answers, all regions were significantly different (Figure 2.a). The next question inquired about the placement of a diverting ileostomy, clinical scenario on an otherwise healthy 52 years old female (BMI $25 \mathrm{Kg} / \mathrm{m} 2)$ who undergoes a laparoscopic LAR for a T2NO rectal cancer a $10 \mathrm{~cm}$ from the anal verge, and the anastomosis below the peritoneal reflection. The most popular answer was NO (39.8\%). Significant differences were identified when comparing SA (48.7\%) against other jurisdictions (ie. vs. EU $[34.7 \%(p<0.05)]$ and OC $[29.1 \%(p<0.05)])$. The highest proportion of a diverting loop ileostomy was reported in OC (39.5\%). All regions were significantly different when comparing the distribution of responses. (Figure 2A)

The acceptable distance to consider a distal margin negative, $>1 \mathrm{~cm}$ was the preferred choice for $46.1 \%$. Only $11.6 \%$ considered that microscopically negative would be appropriate. There was no significant difference among the regions when selecting the top choice nor when assessing the distribution of responses. Question nine presented a 55 year-old male with a posterior T3bN1 lesion at $4 \mathrm{~cm}$ from the anal verge, with evidence of EMVI and apparent complete response after neoadjuvant therapy. The patient was particularly anxious about his cancer prognosis but was also interested in sphincter preservation. The preferred option for the entire group was the performance of a LAR in $58.4 \%$ and the least favorite choice was the use of transanal endoscopic surgery (TES) in $6.8 \%$. The top choice showed significant differences when comparing NA (72\%) against the other regions as follows: EU [56.4\% (p<0.00)]; OC [48.2\% (p<0.001)]; and SA [47 (p< 0.001)]. The response distribution demonstrated differences among all regions except when comparing SA vs OC ( $p>0.05)$. The final question was regarding the use of TNT on a patient with a T2N2 rectal cancer with poor differentiation and evidence of EMVI. This was a binary question, and the majority selected yes (66.50\%). TNT is most popular in Europe (77.6\%), followed by SA (67.6\%) and NA (63.4\%). The most statistically significant differences were noted when comparing EU vs NA [77.6\% vs $63.4 \%$ (p<0.001)], OC vs SA [43.5\% vs 67.6\% (p<0.001)], and NA vs OC [63.4\% vs 43.5\% ( $p<0.001)]$. There was no significant difference between EU and SA 77.6\% vs $67.6 \%$ ( $p>0.05)$. As per the question distribution all regions were statistically different except EU vs SA ( $p>0.05)$. 


\section{Discussion}

To our knowledge, this is the first attempt to contrast therapeutic preferences among rectal cancer surgeons across a number of geographical regions. We identified similarities among regions regarding the top choices, as well as significant differences related to response distribution. We established important variations based on population size (Figure 1).

There is wide access to scientific information regarding the management of rectal cancer \{Dayal:2017fya, Lawson:2019uk\}. However, there are pervasive inequalities regarding the access to resources and technology \{Alkire:2015vw, Newton:2020td\}. We intended to produce a snapshot of the differences in the management of these patients in several regions, by approaching surgeons registered with established colorectal societies. There have been previous attempts to determine the heterogeneity of medical care for rectal cancer patients within countries or limited regions \{Qaderi:2020cg, Crawford:2018cl, Lee:2019je\}, including comparison of outcomes for patients with colorectal malignancies \{Araghi:2019jr\}.

Patterns of presentation of colorectal malignancies vary around the world $\{$ Arnold:2017hi\}. As well there are social and financial differences impacting health care delivery, For instance lack of access is minimal in Australasia, high-income North America and Western Europe \{Alkire:2015vw\}, conversely, in low and middle-income countries, treatment for cancer is not widely available (REF access to cancer treatment). Furthermore, the latter is also identified within socio-economically disadvantaged groups and under-served communities in high-income countries REF(Disparities). The ability to understand and identify the differences regarding the decision making for therapeutic options could help guide outcome-improvement, based on resource availability. It would be imperative to advance global coverage of surgical access \{Alkire:2015vw, Peck:2019vb\}.

EU has the lowest acceptance of low volumes per surgeon or institutions compared to other regions. The definition of "high volume" seemed to have geographic variation and has been long debated as it can be perceived by some as a surrogate of quality (REF Defining the volume). 
We probed the group on its general preference regarding the concept of W\&W, which in general remains a very controversial issue although surgeons and healthcare teams have progressively accepted its value. \{Dossa:2017hh, On:2018de\}. In SA, almost 40\% of participants reported W\&W as the standard option. This is not surprising given that some of the most robust studies originated in Brazil \{HabrGama:2013kc\} (REF nonoperative). Extending the intervals after neoadjuvant therapy has demonstrated improved clinical response (REF Lyon R-96, Probst). Further in the survey, we provided an scenario, depicting a patient with apparent complete clinical response but with evident risk factors for failure and personal preference that might be conflicting. The latter shows how the decision making of $W \& W$, whether on curative or palliative intention, which modality of radiotherapy opt for, is becoming much more complex with increasing availability of different regime and modalities, including endoluminal radiation boosting to increase complete response rates (at the cost of higher long-term toxicity) \{Rullier:2020bh\}\{SunMyint:2019bs\}\{Rijkmans:2019dc\}. The acceptance of the complexities in the management of rectal cancer patients can be linked with some of the other queries we explored in the survey. The majority of cases in EU will be presented at a MDTC; in contrast, almost a quarter of these cases in NA and SA will be discussed at the discretion of the treating physician. This difference is perhaps owing to the longer history in UK and Europe of evolving multidisciplinary approach following the Calman-Hine report in 1995 which showed wide variations in outcome and poor survival of colorectal cancer. Coupled with the fact that healthcare systems in Europe are generally universally accessible and publicly funded, the implementation of multidisciplinary approach has been mandated \{Keller:2020iv\}. It is expected that the role of multidisciplinary team become more important due to the intricated nature of decision making and from governance perspective, considering its proven benefits to patients outcomes (REF Multidisciplinary Obias).

TME has become the standard of surgical care for rectal cancer patients (REF Heald). The principles of TME surgery, as described by Professor Heald can be equally applied to laparoscopic, transanal and robotic TME (REF evolution of surgical treatment) 
The development of the above techniques has aimed to decrease the technical difficulties of performing TME, which represent a formidable challenge in some particular type of patients, e.g obese males \{Kawada:2018da\}. Our survey identified laparoscopic approach as the favorite option on these patients. We noticed the high penetration of robotic surgery in NA, where over $50 \%$ of surgeons consider it for obese male patients. TaTME is almost 3 times more popular in EU than it is in NA and almost twice as popular than in OC. These approaches have technical and ergonomic advantages, increasing the chances of obtaining an optimal TME specimen in difficult cases (REF evolution of surgical treatment). Differences in resources availability may play a major role in geographic penetration and adoption of robotic and taTME techniques which may be reflected in our results.

Diverting ileostomies are not free of complications \{Rathnayake:2008kl\} and in this context, forty percent of participants would be willing to forego its creation on patients with low risk of anastomotic leak. According to our results, participants in OC are more likely to install a diverting ileostomy than in any other region. A ghost or virtual ileostomy \{Baloyiannis:2019jy\} seems to be rarely used, and was only considered by a few European surgeons. Lastly the creation of an ileostomy was at the discretion of the surgeon, solely based on the provided scenario without any discussion of the attendant risks of anastomotic leak, or consultation of the risks of a permanent stoma.

There is great heterogeneity when selecting an acceptable distance for a distal margin. Respondents from OC were more willing to accept a sub-centimeter margin. An APR is more likely to be offered by surgeons from EU when dealing with distal tumors. In contrast, surgeons from other regions would be more likely to choose some form of sphincter sparing approach. The novel concept of TNT has the lowest penetration at this point in $\mathrm{OC}$ where most participants would not consider this as their preferred option, it is however regularly used in NA. TNT has been included and endorsed by the National Comprehensive Cancer Network (NCCN) \{Cercek:2018bd\} .

Additional data will be forthcoming from many of the currently ongoing phase III trials exploring the nonoperative management of rectal cancer after using TNT strategies, their results are 
expected to enhance understanding and applicability of this approach (REF

https://clinicaltrials.gov/ct2/show/NCT02514278) REF preliminary results Organ preservation OPRA-, REF https://clinicaltrials.gov/ct2/show/NCT02505750 (OPERA).

In some questions the most popular response may not be significantly different amongst regions, nonetheless the overall pattern of responses for each region may differ substantially (Figure 2B). For instance, in question number 2 , the preferred choice was similar, but response distribution was different among all regions; this may be important from a clinical standpoint. A plausible explanation: 50\% of surgeons from all regions may choose the same approach (hence, no statistical difference for the top answer), the other $50 \%$ may be very disperse with their choice. The overall approach to surgery in each region is not the same, even though surgeons within each geographical area tend to agree with the choice accepted as the best approach (Figure 2). Differences between Europe/UK and North America may be due to the more mature centers of excellence programs in Europe. Moreover, the single payer systems are more amendable to patient centralization, allowing high volume expert teams to develop.

Limitations include selection bias, since our study is unlikely to be fully representative of the global surgical community taking care of the rectal cancer population; it only considered surgeons registered to colorectal surgical societies and excluded those from regions with low number of respondents, furthermore the lack of participation from countries in Asia or Africa impacts global generalizability, however it is important to mention that a number of surgical societies, in different locations where English is not primarily spoken, did not engage with the initial request to distribute the survey. Conversely, the main advantage of the survey lies on its ability to capture a large sample of surgeons from different regions. Our results provide a unique picture of the variation in the tendencies management of rectal cancer at a multinational level.

\section{Conclusions}

The management of rectal cancer has evolved into a very complex situation, nonetheless there is important variability among surgeons from professional colorectal societies in different countries regarding the perception and ability to deliver treatment to patients with rectal cancer. 
Sociocultural factors and differences in access to resources can possibly explain most of the discrepancies. Further research could provide opportunities to improve care across regions.

\section{Conflict Declaration}

The authors declare no conflict of interest

Funding

No funding was required for this manuscript

Acknowledgments

References

1. Dayal S, Battersby N, Cecil T. Evolution of Surgical Treatment for Rectal Cancer: a Review. J Gastrointest Surg. Springer US; 2017 Apr 25;21(7):1166-73.

2. Heald RJ, Husband EM, Ryall RDH. The mesorectum in rectal cancer surgery-the clue to pelvic recurrence? Br J Surg. John Wiley \& Sons, Ltd; 1982 Oct;69(10):613-6.

3. Kapiteijn E, Marijnen CAM, Nagtegaal ID, Putter H, Steup WH, Wiggers T, et al. Preoperative Radiotherapy Combined with Total Mesorectal Excision for Resectable Rectal Cancer. N Engl J Med. 2001 Aug 30;345(9):638-46. 
4. Sauer R, Becker H, Hohenberger W, Rödel C, Wittekind C, Fietkau R, et al. Preoperative versus Postoperative Chemoradiotherapy for Rectal Cancer. N Engl J Med. 2004 Oct 21;351(17):173140.

5. Allaix ME, Fichera A. Modern Rectal Cancer Multidisciplinary Treatment: The Role of Radiation and Surgery. Ann Surg Oncol. 2013 Apr 21;20(9):2921-8.

6. Bujko K, Nowacki MP, Nasierowska-Guttmejer A, Michalski W, Bebenek M, Kryj M, et al. Long-term results of a randomized trial comparing preoperative short-course radiotherapy with preoperative conventionally fractionated chemoradiation for rectal cancer. Br J Surg. 2006;93(10):1215-23.

7. McQuade RM, Stojanovska V, Bornstein JC, Nurgali K. Colorectal Cancer Chemotherapy: The Evolution of Treatment and New Approaches. CMC. 2017 Jun 30;24(15):1-2.

8. Brouwer NPM, Bos ACRK, Lemmens VEPP, Tanis PJ, Hugen N, Nagtegaal ID, et al. An overview of 25 years of incidence, treatment and outcome of colorectal cancer patients. Int $\mathbf{J}$ Cancer. 2018 Sep 29;143(11):2758-66.

9. Lawson EH, Melvin JC, Geltzeiler CB, Heise CP, Foley EF, King RS, et al. Advances in the management of rectal cancer. Current Problems in Surgery. 2019 Nov;56(11):100648-22.

10. Smith CA, Kachnic LA. Evolving Treatment Paradigm in the Treatment of Locally Advanced Rectal Cancer. J Natl Compr Canc Netw. 2018 Jul 13;16(7):909-15.

11. Lawson E, Melvin J, Geltzeiler C, Heise C, Foley E, King R, et al. Advances in the management of Rectal Cancer. Current Problems in Surgery. 2019 Aug;:1-65. 
12. Glynne-Jones R, Wyrwicz L, Tiret E, Brown G, Rödel C, Cervantes A, et al. Rectal cancer: ESMO Clinical Practice Guidelines for diagnosis, treatment and follow-up $†$. Annals of Oncology. 8 ed. 2017 Jul 13;28(suppl_4):iv22-iv40.

13. Dossa F, Chesney TR, Acuna SA, Baxter NN. A watch-and-wait approach for locally advanced rectal cancer after a clinical complete response following neoadjuvant chemoradiation: a systematic review and meta-analysis. The Lancet Gastroenterology \& Hepatology. 2017 $\mathrm{Jul} ; 2(7): 501-13$.

14. Fleshman J, Branda M, Sargent DJ, Boller AM, George V, Abbas M, et al. Effect of LaparoscopicAssisted Resection vs Open Resection of Stage II or III Rectal Cancer on Pathologic Outcomes. JAMA. American Medical Association; 2015 Oct 6;314(13):1346-10.

15. Marks J, Mizrahi B, Dalane S, Nweze I, Marks G. Laparoscopic transanal abdominal transanal resection with sphincter preservation for rectal cancer in the distal $3 \mathrm{~cm}$ of the rectum after neoadjuvant therapy. Surgical Endoscopy. 2010 Apr 23;24(11):2700-7.

16. Penna M, Buchs NC, Bloemendaal AL, Hompes R, Penna M, Buchs NC, et al. Transanal total mesorectal excision for rectal cancer: The journey towards a new technique and its current status. Expert Review of Anticancer Therapy. Taylor \& Francis; 2016 Sep 21;0(0):1-35.

17. Baik SH, Kang CM, Lee WJ, Kim NK, Sohn SK, Chi HS, et al. Robotic total mesorectal excision for the treatment of rectal cancer. J Robotic Surg. Springer-Verlag; 2007 Feb 10;1(1):99-102.

18. Cercek A, Roxburgh CSD, Strombom P, Smith JJ, Temple LKF, Nash GM, et al. Adoption of Total Neoadjuvant Therapy for Locally Advanced Rectal Cancer. JAMA Oncol. 2018 Jun 1;4(6):e180071-16. 
19. Allaix ME, Fichera A. Modern Rectal Cancer Multidisciplinary Treatment: The Role of Radiation and Surgery. Ann Surg Oncol. 2013 Apr 21;20(9):2921-8.

20. Wexner SD, Berho ME. The Rationale for and Reality of the New National Accreditation Program for Rectal Cancer. Diseases of the Colon \& Rectum. Diseases of the Colon \& Rectum; 2017;60(6):595-602.

21. Qaderi SM, Wijffels NAT, Bremers AJA, de Wilt JHW. Major differences in follow-up practice of patients with colorectal cancer; results of a national survey in the Netherlands. BMC Cancer. 2020 Jan 6;20(1):2758-8.

22. Dayal S, Battersby N, Cecil T. Evolution of Surgical Treatment for Rectal Cancer: a Review. J Gastrointest Surg. Springer US; 2017 Apr 25;21(7):1166-73.

23. Lawson E, Melvin J, Geltzeiler C, Heise C, Foley E, King R, et al. Advances in the management of rectal cancer. 2019 Aug 15;:1-65.

24. Alkire BC, Raykar N, Shrime MG, Weiser T, Bickler S, Rose J, et al. Global access to surgical care: a modelling study. The Lancet. 2015 Jun;3:316-23.

25. Newton P, Bond K. Comment Global access to quality-assured medical products: the Oxford Statement and call to action. The Lancet. 2020 Feb 17;7:e1609-11.

26. Crawford A, Firtell J, Caycedo-Marulanda A. How Is Rectal Cancer Managed: a Survey Exploring Current Practice Patterns in Canada. J Gastrointest Canc. Springer US; 2018 Feb 1;10(3):219-20.

27. Lee H, Kung P, Wang Y, Juo W, Kao L, Tsai W. Effect of length of time from diagnosis to treatment on colorectal cancer survival: A population-based study. PLoS ONE. 2019 Jan 14;:1-16. 
28. Araghi M, Soerjomataram I, Bardot A, Ferlay J, Cabasag CJ, Morrison DS, et al. Changes in colorectal cancer incidence in seven high-income countries: a population-based study. The Lancet Gastroenterology \& Hepatology. 2019 May 16;4(7):511-8.

29. Arnold M, Sierra MS, Laversanne M, Soerjomataram I, Jemal A, Bray F, et al. Global patterns and trends in colorectal cancer incidence and mortality. Gut. BMJ Publishing Group; 2017 Apr;66(4):683-91.

30. Peck G, Roa L, E B, South S, Foainini E, Vaz-Ferreira R, et al. Improving global emergency and essential surgical care in Latin America and the Caribbean- A collaborative approach. Bulletin American College of Surgeons. 2019 Mar 1;304(3):24-39.

31. On J, Aly EH. "Watch and wait" in rectal cancer: summary of the current evidence. International Journal of Colorectal Disease. 2018 Jul 5;33(9):1159-68.

32. Habr-Gama A, Sabbaga J, Gama-Rodrigues J, São Julião GP, Proscurshim I, Bailão Aguilar P, et al. Watch and wait approach following extended neoadjuvant chemoradiation for distal rectal cancer: are we getting closer to anal cancer management? Diseases of the Colon \& Rectum. 2013 Oct;56(10):1109-17.

33. Kawada K, Sakai Y. Can we predict surgical difficulty of rectal surgery? Ann Laparosc Endosc Surg. 2018 May;3:44-4.

34. Rathnayake MMG, Kumarage SK, Wijesuriya SRE, Munasinghe BNL, Ariyaratne MHJ, Deen KI. Complications of loop ileostomy and ileostomy closure and their implications for extended enterostomal therapy: A prospective clinical study. International Journal of Nursing Studies. 2008 Aug;45(8):1118-21. 
35. Baloyiannis I, Perivoliotis K, Diamantis A, Tzovaras G. Virtual ileostomy in elective colorectal surgery: a systematic review of the literature. Tech Coloproctol. 2019 Dec 9;24(1):23-31.

36. Kellerman S, Herold J. Physician Response to Surveys. Am J Prev Med. 2001;20(1):61-7.

\section{Appendix A}

\section{Questions}

1. What is the minimum annual number of TME operations to be considered a high volume rectal cancer surgeon?
a. $>10$
b. $>20$
c. $>40$
d. $>50$

2. What is the minimum annual number of rectal cancers operated on to be considered a high volume rectal cancer centre?
a. $>20$
b. $>40$
c. $>80$
d. $>100$

3. Which rectal cancer cases should be presented at multidisciplinary conference?
a. All
b. Only advanced stages
c. As per discretion of the treating surgeon/oncologist
d. None

4. Do you include watch \& wait as part of your practice
a. Yes, it is standard at my center
b. Never (Not standard)
c. Only as per patient request
d. Poor surgical candidates 
5. 57 yo female had a poorly differentiated rectal cancer at $4 \mathrm{~cm}$, she underwent a long course of chemoradiation after 8 weeks she seems to have developed a complete clinical response, what option would you consider for her?
a. Carry on with LAR as planned
b. Offer to enrol her on a W\&W protocol
c. Offer Transanal endoscopic surgery (TES)
d. Send her for a second opinion

6. A 65 yo obese male (BMI 35) has a T2N0 rectal cancer at $6 \mathrm{~cm}$ from the anal verge, without adverse features. What surgical approach would you choose for this case in your practice
a. Open LAR
b. Laparoscopic LAR
c. Robotic LAR
d. TaTME LAR

7. A 52 yo female (BMI 25) undergoes a laparoscopic LAR for a $T 2 N 0$ rectal cancer a $10 \mathrm{~cm}$ from the anal verge (No neoadjuvant therapy). Anastomosis is below peritoneal reflection. Would you create a diverting ileostomy?
a. Yes
b. No
c. Only if leak test is $(+)$
d. Ghost ileostomy

8. What is the minimum acceptable distal margin following TME?
a. Microscopically negative
b. $1-2 \mathrm{~mm}$
c. $>2 \mathrm{~mm}$
d. $>1 \mathrm{~cm}$

9. A 55 yo male with a posterior $T 3 b N 1$ rectal $C A$ at $4 \mathrm{~cm}$ from the anal verge, with evidence of EMVI, apparent complete response after neoadjuvant therapy is suggested. He is very anxious about his cancer prognosis but is also interested in sphincter preservation. What option would you recommend?
a. Low Anterior resection
b. Watch \& Wait protocol
c. Transanal endoscopic resection (TEMS/TAMIS)
d. Abdominoperineal resection 
10. In a 54 yo male with a T2 N2 poorly differentiated mid rectal cancer with evidence of EMVI on MRI would total neoadjuvant therapy (TNT) rather than split chemoradiation be considered as an option in your practice?
a. Yes
b. No 